\title{
Sensibilité de la production herbacée aux aléas de la distribution des pluies au Sahel (Agoufou, Mali): une approche par modélisation
}

\author{
Mamadou Oumar DIAWARA ${ }^{1 *}$, Pierre HIERNAUX ${ }^{2}$, Sory SISSOKO ${ }^{1}$, Eric MOUGIN $^{3}$, \\ Alassane BA $^{4}$, Nogmana SOUMAGUEL ${ }^{5}$ et Hawa Salif DIAKITÉ 6 \\ ${ }^{1}$ Département de Biologie, Faculté des Sciences et Techniques (FST), Université des Sciences, des Techniques \\ et des Technologies de Bamako (USTTB), Colline de Badalabougou, B.P. 3206 Bamako, Mali. \\ ${ }^{2}$ Hiernaux, Pierre, Pastoc, 30 chemin de Jouanal, 82160 Caylus, France. \\ ${ }^{3}$ Géosciences Environnement Toulouse (GET), Observatoire Midi-Pyrénées, Université de Toulouse, CNRS, \\ IRD, 14 avenue Edouard Belin, 31400 Toulouse Cedex-France. \\ ${ }^{4}$ Institut d'Economie Rurale (IER), Centre Régional de Recherche Agronomique de Sikasso (CRRA), \\ Programme Bovin, BP. 16 Sikasso, Mali. \\ ${ }^{5}$ Centre IRD de Bamako, Quartier Hippodrome, BP 2528 Bamako, Mali. \\ ${ }^{6}$ Institut d'Economie Rurale (IER), Centre Régional de Recherche Agronomique de Sotuba (CRRA), \\ Laboratoire Sol-Eau-Plante, Unité Système d'Information Géographique et Télédétection, B.P. 262 \\ Bamako, Mali. \\ *Auteur correspondant ; E-mail : diaprod@hotmail.com
}

\section{RESUME}

La forte variation du régime des pluies au Sahel est reconnue par tous. La phénologie de la strate herbacée dominée par des plantes annuelles suit ce cycle annuel en faisant alterner une courte saison de croissance et une longue saison morte. Le Gourma situé dans la bande sahélienne au Mali est une zone pastorale soumise à cette variabilité temporelle des ressources pastorales (eau et fourrage) liée au régime de la mousson Ouest africaine, et aux fortes variations interannuelles de la pluviosité. Cette étude a pour objectif d'évaluer la sensibilité de la production de la strate herbacée aux variations de la distribution intra-saisonnière des pluies au Sahel à travers le cas du site pastoral d'Agoufou. Les simulations ont été effectuées avec le modèle STEP (Sahelian Transpiration Evaporation and Production model). Les analyses reposent sur de simples permutations des pluies journalières enregistrées sur le site en 2010, réalisées de façon aléatoire sans modifier le total saisonnier ni les cumuls journaliers. Les résultats montrent que la relation entre le cumul des pluies saisonnières et les phytomasses herbacées mesurées en fin de saison masque des chutes de production intra-saisonnière liées aux stress hydriques, notamment au cours de la période de croissance rapide qui affectent la production saisonnière. Le modèle pourrait servir dans un système intégré d'alertes précoces pour anticiper les pénuries de fourrage fréquentes dans cette région.

(C) 2020 International Formulae Group. All rights reserved.

Mots clés : Production primaire, strate herbacée, variation pluviométrique, stress hydrique, modèle STEP. 


\title{
Sensitivity of herbaceous vegetation production to the variability of rainfall distribution in the Sahel (Agoufou, Mali): a modeling approach
}

\begin{abstract}
The strong spatial and temporal variability of rainfall patterns in the Sahel is accepted by everybody. The phenology of the herbaceous layer dominated by annual plants follows this rhythm by alternating a short growing period with a long dormancy period. The Gourma region located in the Sahelian belt in Mali is a pastoral area subject to this temporal variability of resources (water and forage) linked to the rains regime. This study aims to assess the sensitivity of the herbaceous vegetation production to the variation of the intra-seasonal rainfall distribution in the Sahel through the case of the Agoufou rangeland site. The simulations were performed with the STEP model (Sahelian Transpiration Evaporation and Production model). The analyses were based on simple changes of rainfall height recorded on the site in 2010, carried out randomly without changing the seasonal total or the daily totals. The results show that the relationship between rainfall totals and the fall of the herbaceous mass conceal intra-seasonal production related to water stress especially during the period of so-called main growth can affect the seasonal production. The model could be used in an integrated early warning system to anticipate frequent forage shortages in this region.
\end{abstract}

(C) 2020 International Formulae Group. All rights reserved.

Keywords: Primary production, herbaceous layer, rainfall variation, water deficit, STEP model.

\section{INTRODUCTION}

Le Sahel est connu pour sa forte hétérogénéité spatiale et la variabilité interannuelle de la production primaire de ses parcours liée au régime des pluies (Tracol, 2004). Le régime annuel des précipitations commandé par la mousson Ouest africaine est caractérisé par une distribution des précipitations de juillet à septembre (Frappart et al., 2009) dont le déroulement (date de début, de fin, le volume des pluies, leur distribution, leur intensité) est déterminant pour la production fourragère (Hiernaux et al., 2009). De plus, les contraintes liées aux variations du volume des pluies annuelles sont accentuées par l'hétérogénéité spatiale et la grande variabilité de la distribution des précipitations à l'intérieur de la saison des pluies (Balme et al., 2005). L'incidence des déficits généraux de la pluviosité annuelle sur plusieurs années consécutives peut s'avérer dramatique pour les populations pastorales et leur cheptel, comme au cours des grandes sécheresses de 1972-1973 et 1982-1984 (Hiernaux et al., 2015).

Au Sahel, la phénologie de la strate herbacée largement dominée par les thérophytes (Hiernaux et Le Houérou, 2006) surtout des graminées, suit ce rythme saisonnier en faisant alterner une vigoureuse mais brève saison de croissance à une longue saison morte (Ousseina et al., 2013). Le stock de pailles est constitué pendant la courte période de vie active des pâturages. La quantité produite varie avec les pluies, mais également avec la nature du sol et la situation topographique qui influent sur le régime d'humidité du sol (Hiernaux et al., 2009; Samandoulgou et al., 2019). Ces pailles, ou foins sur pied, constituent, avec l'appoint de fourrages verts apportés par les plantes ligneuses, l'essentiel des ressources fourragères sur lesquelles le bétail sahélien doit compter tout au long des 8 à 10 mois de la saison sèche (Achard et al., 2001 ; Hiernaux et al., 2013).

Le Gourma, région naturelle située dans la bande sahélienne du Mali entre le 15 ème et le $17^{\text {ème }}$ parallèle (Mougin et al., 2009) est une zone essentiellement pastorale soumise à une forte variabilité interannuelle des ressources en eau de surface et en fourrage liée au régime des pluies (Diawara, 2015). Le cumul annuel moyen des précipitations de 1950 à 2007 varie de 100 à 400 mm (Frappart et al., 2009). La 
végétation se présente sous forme d'un tapis herbacé plus ou moins continu composé d'herbacées annuelles et une strate ligneuse très lâche composée d'épineux du genre acacia, de Combrétacées, de Caparidacées et possède une couverture n'excédant pas $5 \%$ sauf dans les dépressions interdunaires (Hiernaux et Le Houérou, 2006).

L'objectif de cette étude est d'évaluer la sensibilité de la production primaire de la strate herbacée à la variation de la distribution intrasaisonnière des pluies au Sahel à travers le cas du site d'Agoufou $\left(15.3^{\circ} \mathrm{N} ; 1.5^{\circ} \mathrm{W}\right)$. Pour ce faire, nous avons utilisés le modèle STEP (Sahelian Transpiration Evaporation and Production model), un modèle de simulation de la croissance de la végétation herbacée au Sahel (Tracol et al., 2006).

\section{MATERIEL ET METHODES}

\section{Présentation du site de l'étude (Agoufou)}

Situé à proximité du village d'Agoufou $(2 \mathrm{~km})$ et de la mare permanente éponyme, le site d'Agoufou est soumis à une forte pression pastorale toute l'année (Diawara et al., 2018). Les sols du site sont sableux en forme de dunes maillées, fixées par la végétation d'herbacée annuelle à l'exception de quelques plages de déflation et d'accumulation dynamiques sur les crêtes et de petits arbres, arbustes et buissons épars. Le relief dunaire est assez marqué avec une dissymétrie caractéristique. La strate herbacée qui s'y développe se compose exclusivement d'espèces annuelles dont les masses mesurées en fin de saison des pluies varient en fonction de la position topographique et surtout d'une année à l'autre. Le recouvrement moyen de la strate herbacée au cours de la période 1984-2002 est estimé à $20 \%$ avec une variation interannuelle de $46,2 \%$ (Tracol, 2004). La composition est dominée par les graminées annuelles (Aristida mutabilis, Cenchrus biflorus, Dactyloctenium aegyptiacum, Tragus berteronianus) mais avec la présence importante localement (et certaines années) de dicotylédones telles que Zornia glochidiata, Tribulus terrestris. Le peuplement ligneux est épars avec une concentration surtout des arbres dans les dépressions interdunaires. Essentiellement d'espèces de la famille des mimosacées (Acacia raddiana, Acacia senegal), d'espèces à feuilles plus grandes, épaisses et cireuses (Combretum glutinosum et Balanites aegyptiaca) et, depuis la sècheresse de 1972-73, s'est installé dans la strate buissonnante une espèce sempervirente mais aphylle (Leptadenia pyrotechnica). Ce site concentrait un grand nombre d'appareils de mesures dont trois pluviographes et faisait l'objet d'un suivi régulier de la dynamique de la végétation naturelle (herbacée et ligneuse) en relation avec la variation interannuelle de la pluviosité (Mougin et al., 2019). Depuis la crise sécuritaire du nord-Mali (2012), le dispositif se limite à quelques pluviomètres implantés dans les villages proches des sites pastoraux dont celui d'Agoufou et le suivi de la végétation ne porte plus que sur la dynamique de la strate herbacée.

\section{Le modèle STEP (Sahelian Transpiration Evaporation Product model)}

Le modèle STEP simule la croissance de la masse herbacée au Sahel. Le modèle comporte deux modules principaux simulant d'une part la production primaire et d'autre part la dégradation de la masse herbacée sur pied au cours de la saison sèche (Delon et al., 2015 ; Diawara et al., 2018). Le compartiment de production de la masse herbacée est constitué de deux modules interdépendants: un module de croissance de la végétation (photosynthèse et respiration) et un module de bilan d'eau (Figure 1). Le code est écrit en langage Fortran. Le modèle tourne à un pas de temps journalier et nécessite en entrée, des variables climatiques journalières (précipitations, température et humidité relative de l'air, rayonnement global, vitesse du vent), de la composition végétale (proportion de plantes en $C 3$ et en $C 4$, proportion de monocotylédones/ dicotylédones) et des caractéristiques du sol (pente, profondeur et texture) et de la charge 
animale. Les paramètres du module STEP sont présentés dans le Tableau 1.

\section{Fluctuations intra-saisonnières des hauteurs de pluies}

Pour appréhender l'empreinte de la mousson sur les fluctuations intra-saisonnières des précipitations dans notre région d'étude et déterminer des variables caractéristiques du déroulement de la saison des pluies, des précipitations journalières enregistrées par les stations pluviométriques de Rharous $\left(16.5^{\circ} \mathrm{N}\right.$; $\left.1.5^{\circ} \mathrm{W}\right)$, d'Agoufou $\left(15.3^{\circ} \mathrm{N} ; 1.5^{\circ} \mathrm{W}\right)$ et de Kobou $\quad\left(14.7^{\circ} \mathrm{N} ; \quad 1.5^{\circ} \mathrm{W}\right)$ situées respectivement à l'extrême nord, au centre et au sud de la région du Gourma ont été analysées. La démarche adoptée a consisté à la détermination des cumuls annuels de pluies puis à la décomposition de l'ensemble des précipitations de la saison en pluies précoces (pluies enregistrées avant le début des pluies utiles), pluies tardives (pluies tombées après la fin des pluies utiles) et en pluies utiles à la croissance de la végétation (ensemble des précipitations de l'année auxquelles sont soustraits les pluies précoces et les pluies tardives). Cette période de croissance rapide de la végétation est déterminée en fonction des dates de démarrage et de fin de la saison des pluies. Les études conduites dans la région sahélienne visant leur détermination se fondent sur trois approches : climatique, hydrologique et agronomique (Balme et al., 2005).

L'approche agronomique a reposé sur l'enregistrement d'au moins $20 \mathrm{~mm}$ de pluies sur 3 jours sans intervention d'une séquence sèche de plus de 7 jours au cours d'un mois d'observation à partir de mi-mai (Balme et al., 2005). La date de démarrage agronomique est autrement définie par les pluies dites significatives qui permettent le déclenchement des premières germinations. Une période sans pluies de 20 jours consécutifs au-delà de miaoût marque la fin de la saison des pluies (Balme et al., 2005). La détermination des dates de démarrage et de fin de la saison des pluies mais aussi celle de toutes les autres variables présentées antérieurement s'est appuyée sur cette approche agronomique.

\section{Analyses de sensibilité}

La sensibilité de la production primaire herbacée à la variation de la distribution intrasaisonnière des hauteurs de pluies a été évaluée à l'aide du modèle STEP. L'analyse repose sur de simples permutations des hauteurs de pluies quotidiennes enregistrées sur le site pastoral d'Agoufou en 2010, réalisés de façon aléatoire sans modifier le total saisonnier.

Ces tirages aléatoires avec remise ont été effectués dans un premier temps sur les hauteurs de pluies enregistrées sur la série complète (tous les évènements pluvieux de la saison). Ces tirages ont pour but de faire varier la distribution intra-saisonnière des hauteurs de pluies sans modifier le total saisonnier et d'observer leurs effets sur la production estimée par la phytomasse maximale en fin de saison. Nous avons ainsi analysé les productions de phytomasse herbacée de 30 simulations. La production a été également analysée pour chaque période de la croissance végétale: germination, installation-tallage, montaison, floraison-fructification, mort.

Tableau 1 : Principaux paramètres du modèle STEP et références associées.

\begin{tabular}{lcc}
\hline Paramètres & Valeurs & Sources \\
\hline Biomasse initiale $\left(\mathrm{B}_{\mathrm{g}} 0\right)$ & $1 \mathrm{~g} \mathrm{MS} / \mathrm{m}^{2}$ & Pierre et al., 2011 \\
Specific Leaf Area initial (SLA 0$)$ & $200 \mathrm{~cm}^{2} / \mathrm{g}$ & Jarlan et al., 2008 \\
Proportion des plantes en $C 3$ et en $C 4$ & $43 \%$ et $57 \%$ & Hiernaux et al., 2009 \\
\hline
\end{tabular}




\section{Entrées}

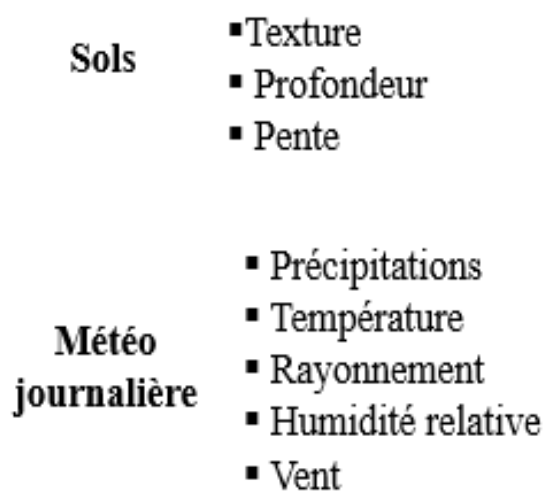

$\begin{array}{ll}\text { Végétation } & \text { Proportion de } \mathrm{C} 3 / \mathrm{C} 4 \\ & \text { - Mode de dispersion }\end{array}$
STEP

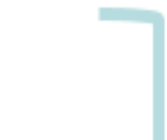

Bilan Hydrique

Végétation racinaire)

LAI

Recouvrement

Hauteur

Sorties

Contenu en eau du sol Evaporation Transpiration Ruissellement

Phytomasse (aérienne et ment

Figure 1 : Représentation schématique du module de production primaire de la masse herbacée (adapté de Mougin et al., 1995).

\section{RESULTATS}

\section{Distribution intra-saisonnière des pluies}

La Figure 2 présente les cycles saisonniers moyens des pluies à partir des précipitations journalières enregistrées à Rharous au Nord du Gourma aux abords du fleuve Niger, à Agoufou au centre de la région et à Kobou au sud du Gourma pour la période 2004-2010.

La saison des pluies est plus courte à Rharous où elle dure en moyenne 46 jours contre 70 et 76 jours respectivement à Agoufou et à Kobou. A Kobou, la saison des pluies démarre en moyenne autour du 19 Juin et prend fin autour du 3 septembre alors qu'à Rharous, le démarrage a lieu vers le 4 juillet pour une fin de saison autour du 19 août. Les cumuls annuels moyens de précipitations au cours de la période considérée sont de 158,$83 ; 346,51$ et $358,84 \mathrm{~mm}$ respectivement à Rharous, à Agoufou et Kobou. Mais tout au long du gradient, les cumuls annuels de précipitations aussi bien que les longueurs des cycles saisonniers sont marqués d'une forte variabilité interannuelle. Pour la durée de la saison des pluies, le coefficient de variation est plus élevé à Rharous $(69,40 \%)$, au nord, qu'au centre à Agoufou $(50,43 \%)$ et au sud du Gourma à Kobou (40,18\%), alors que la variabilité des cumuls annuels de précipitations, est plus élevée à Agoufou (32,4\%) qu'à Rharous $(22,9 \%)$ et à Kobou $(31 \%)$. Tout le long du gradient bioclimatique sahélien, la distribution des pluies est centrée autour du mois d'août avec des évènements pluvieux d'avant et d'après installation de la mousson (évènements survenus avant le 1 mai pour les pluies précoces et après le 31 octobre pour les pluies tardives). Ces évènements se définissent par rapport aux précipitations considérées comme utiles au développement végétal, et ils définissent à leur tour la période utile à la croissance principale des plantes annuelles (cultures pluviales et les herbacées annuelles). 
L'analyse de la distribution des pluies indique que seuls $18,7 \%\left(29,8 \mathrm{~mm} \mathrm{an}^{-1}\right)$ des cumuls annuels moyens de précipitations sont utiles à la croissance rapide de la végétation à Rharous alors qu'à Kobou et à Agoufou, les pluies utiles représentent respectivement $36,8 \%(124,4 \mathrm{~mm}$ $\left.\mathrm{an}^{-1}\right)$ et $42,5 \%\left(131,1 \mathrm{~mm} \mathrm{an}^{-1}\right)$ des cumuls moyens annuels.

\section{Variation interannuelle de la production primaire de la strate herbacée}

Les productions primaires de phytomasse herbacée mesurées en fin de saison de croissance sur le site pastoral d'Agoufou entre 2002 et 2011, sont présentées dans la Figure 3. La production moyenne de phytomasse aérienne au cours de cette période est de 158,21 $\mathrm{g} \mathrm{MS} \mathrm{m}^{-2}$. L'analyse de la variation de cette production au cours de la période, montre de grandes disparités $(39,01 \%$ de variation). Les productions annuelles de phytomasse herbacée d'un même site sont du simple au quadruple comme le montre la différence de production entre les années 2004 et 2010 .

La saison de production de la végétation herbacée démarre dans la première décade du mois de juillet autour du jour 188 (7 juillet) pour atteindre le maximum de végétation autour du jour 255 (12 septembre). La phase de croissance rapide de cette végétation s'étale sur 68 jours en moyenne. Cette première analyse montre que la production herbacée dépend beaucoup de la distribution des pluies au cours de la saison.

\section{Sensibilité de la production de la strate herbacée aux aléas de la distribution intra- saisonnière des pluies à Agoufou}

La distribution observée et les deux scenarios présentant les extrêmes de production sont présentés dans la Figure 4. Les figures de gauche ( $4 \mathrm{a}, 4 \mathrm{~b}$ et $4 \mathrm{c})$ présentent les variations saisonnières des réserves hydriques simulées dans la couche racinaire $(2-30 \mathrm{~cm})$ pour la distribution observée et les deux scenarios. Celles de droite (4A, 4B et 4C), les productions de phytomasse correspondantes.

Plus que l'importance des évènements pluvieux, la production de masse dépend de la répartition des séquences sèches au cours de la saison des pluies. Dans la disponibilité la moins régulière de l'échantillon (Figure 4B), la première séquence sèche (17 jours) coïncide avec la phase sensible de la croissance principale dans le modèle et génère un déficit de production de $42,4 \%$ par rapport à la distribution observée (Figure 4A).

Dans le meilleur scénario, la distribution génère une production maximale supérieure à celle de la distribution observée de 18,9\% (Figure 4C). Dans la distribution observée, les pluies de juillet représentent $36,3 \%$ du cumul annuel alors que dans la distribution la moins régulière, elles ne représentent que $11,8 \%$ du cumul annuel. On peut supposer que les pluies enregistrées au cours de cette période jouent un rôle dans la croissance principale de la végétation herbacée et la production de matière sèche. Par ailleurs, on constate qu'à cumul pluviométrique égal, la production de la végétation herbacée dépend en partie de la longueur de la période de croissance rapide. L'analyse effectuée à partir des scénarios de distribution des pluies montre que cette période de croissance rapide détermine pour $61,4 \%$, la variation intrasaisonnière de la production aérienne de la strate herbacée.

Les tests de sensibilité montrent que près de $73,3 \%$ des productions de phytomasse aérienne simulées par le modèle sont supérieures à $200 \mathrm{~g} \mathrm{MS} \mathrm{m}^{-2}$ (Figure 5). La production maximale se situe entre 250 et 300 g MS $\mathrm{m}^{-2}$ alors même que la phytomasse maximale mesurée sur le site d'Agoufou en 2010 est de 243,3 $\mathrm{g} \mathrm{MS} \mathrm{m}^{-2}$. 
Rharous 2004 - 2010 (158.83 mm)

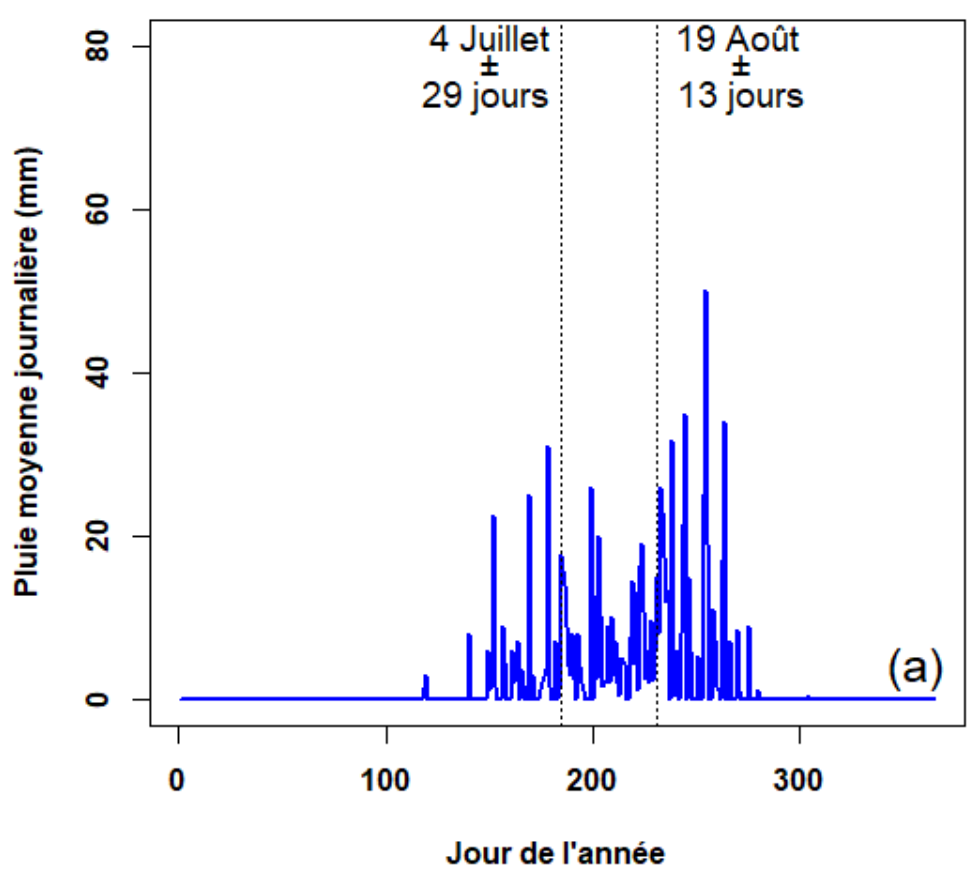

Agoufou 2004 - 2010 (346.51 mm)

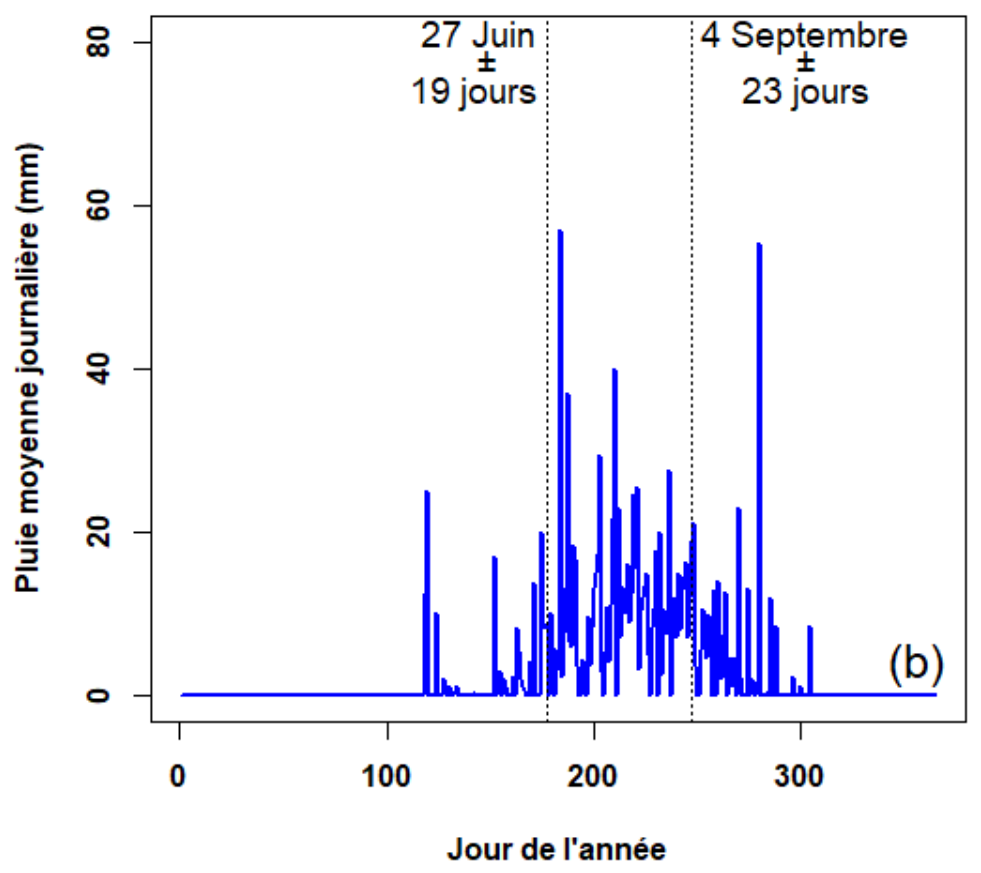




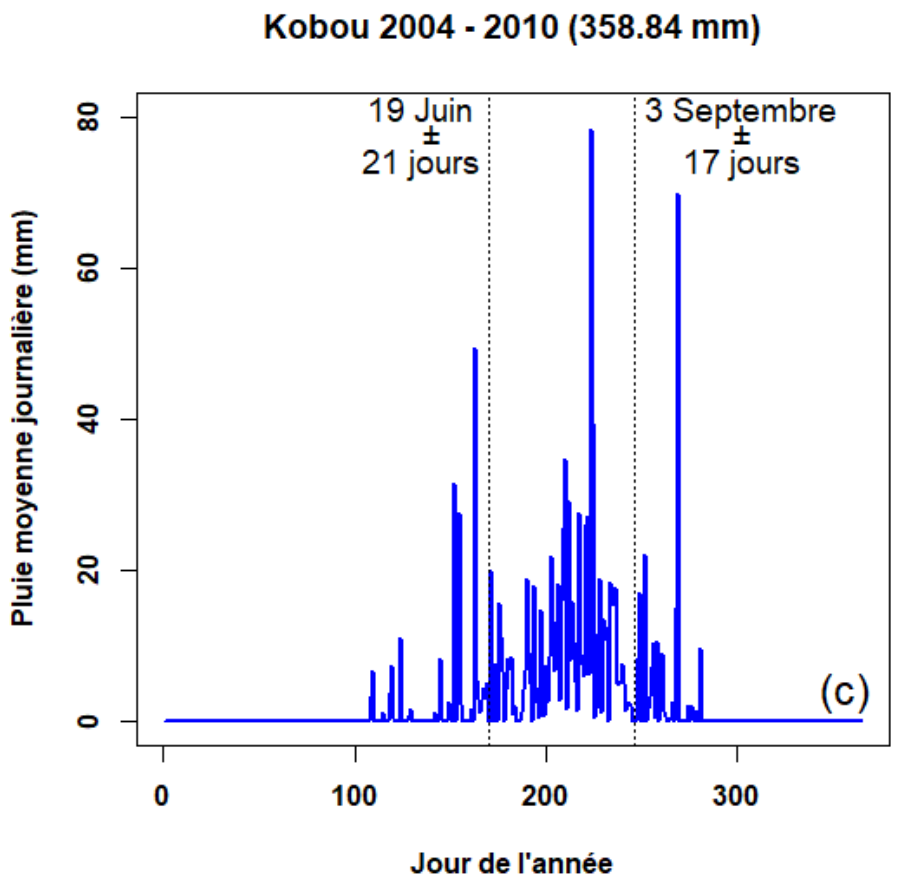

Figure 2 : Cycles saisonniers moyens des pluies à partir des précipitations journalières des postes pluviométriques de Rharous (a), d'Agoufou (b) et de Kobou (c) entre 2004 et 2010. Les dates de début et de fin des pluies sont portées sur chaque graphe.

Agoufou $\left(15.3^{\circ} \mathrm{N}, 1.5^{\circ} \mathrm{W}\right)$

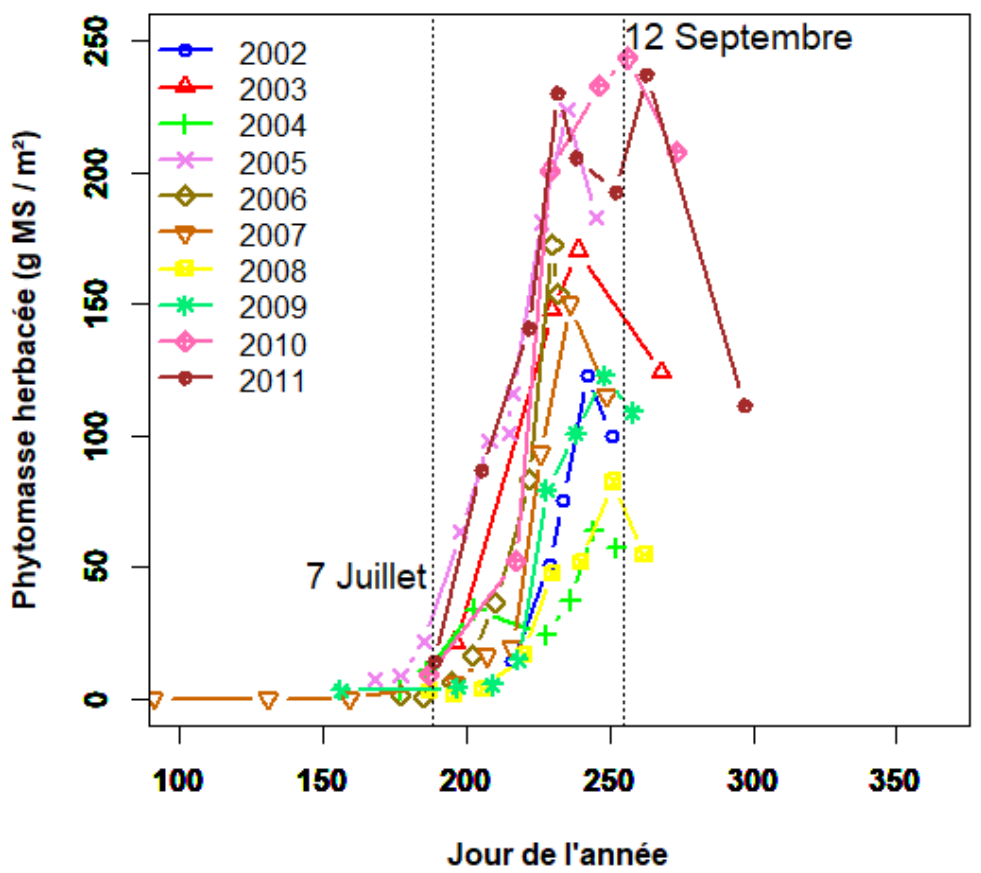

Figure 3 : Variation temporelle de la masse herbacée aérienne sur le site pastoral d'Agoufou au cours de la saison de croissance. 

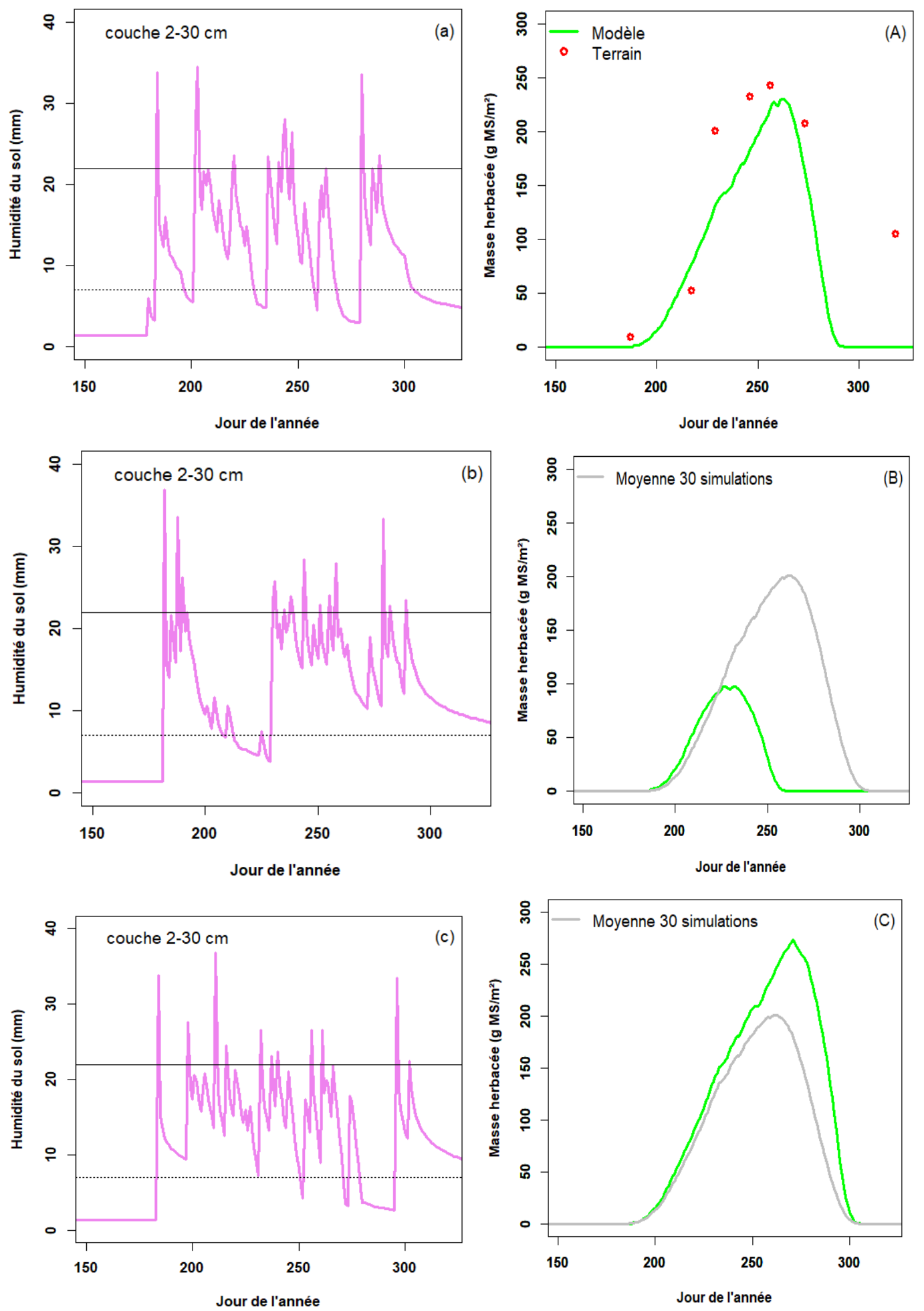

Figure 4: Courbe des masses herbacées correspondantes aux scénarios de distributions intrasaisonnières de pluies sur le site pastoral d'Agoufou. Les figures de gauche (a, b et c) présentent les variations saisonnières des réserves hydriques simulées dans la couche racinaire $(2-30 \mathrm{~cm})$ de la situation observée et des deux scenarios. Les figures de droite ( $\mathrm{A}, \mathrm{B}$ et $\mathrm{C}$ ), les masses herbacées aériennes correspondantes. 


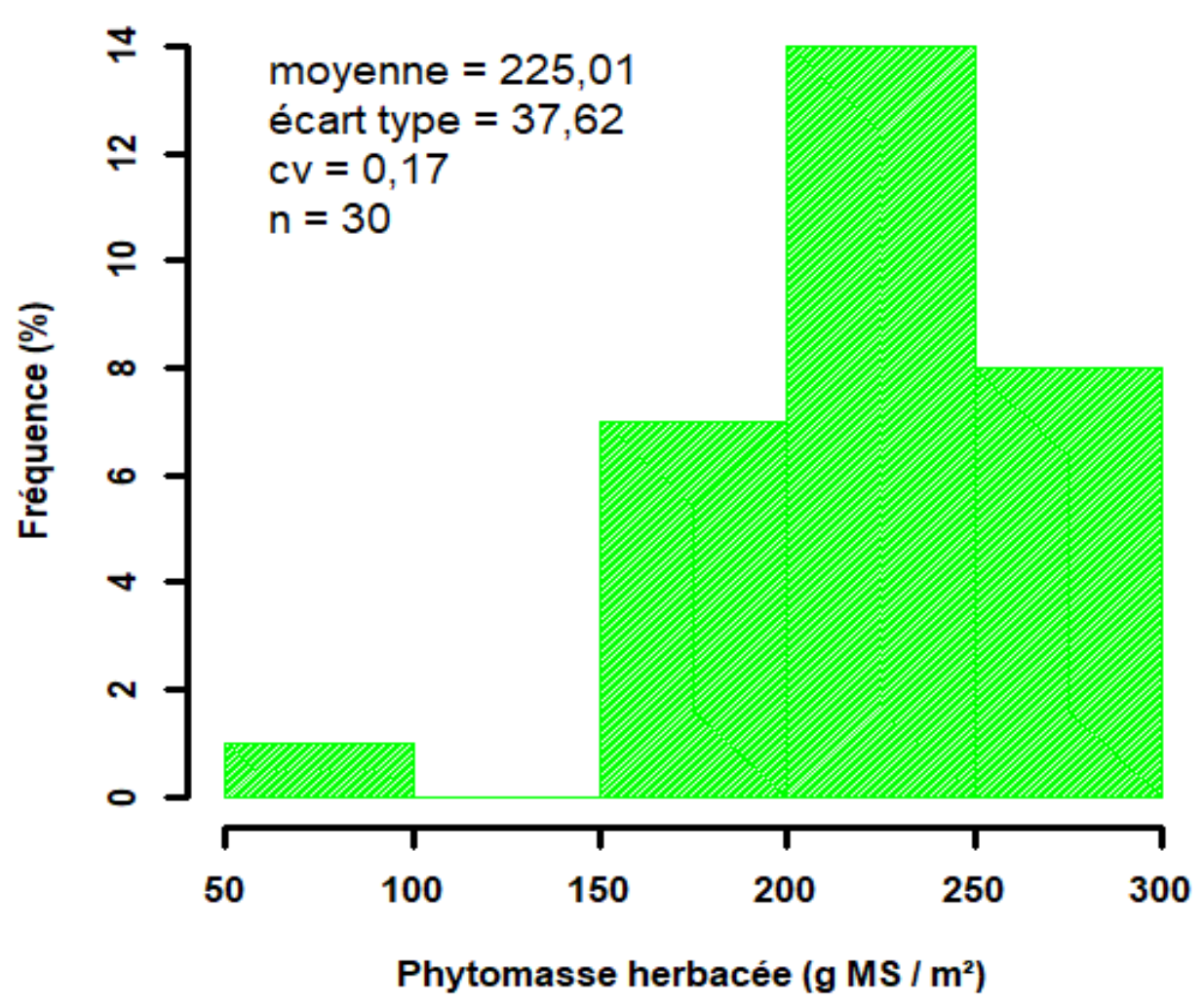

Figure 5 : Distribution des productions aériennes herbacées de 30 simulations par permutation aléatoire des pluies journalières de la saison des pluies observée à Agoufou en 2010.

\section{DISCUSSION}

La production primaire de la strate herbacée apparait particulièrement sensible à la distribution des pluies au cours de la phase de montaison qui correspond au mois de juillet à Agoufou en 2010. Les analyses établissent une relation entre la production aérienne herbacée et la durée de la saison de croissance qui explique $60 \%$ de sa variation selon les scenarios de permutation des pluies sur la saison. Elles montrent que la récurrence des déficits de production primaire de la strate herbacée est proportionnelle à l'amplitude de variation de la répartition des hauteurs de pluies. La production est limitée par les contraintes hydriques et radiatives fixées dans le modèle (Tracol et al., 2006 ; Pierre et al., 2011).
Les dates de démarrage et de fin de la saison des pluies ont une faible incidence sur la production aérienne de phytomasse herbacée. Dans les deux cas, les précipitations enregistrées au cours du mois de juillet semblent jouer un grand rôle sur la production saisonnière de la strate herbacée.

La relation entre les phytomasses maximums et les cumuls de pluie annuels montre que pour des précipitations très variables dans le temps, les cumuls de pluie annuels expliquent $74 \%$ des variations interannuelles de la production primaire de la strate herbacée mesurée de 2002 à 2011 sur le site pastoral d'Agoufou. Des études antérieures effectuées sur ces relations au Sahel (Tracol, 2004 ; Hiernaux et al., 2009), vont dans le même sens en montrant les effets de facteurs 
externes (pâture et la fertilité du sol) au déroulement de la saison des pluies sur la production de phytomasse en région sahélienne.

Ces analyses ne prennent pas en compte l'influence du peuplement ligneux sur la variation de la production de la strate herbacée ni les caractéristiques de la végétation notamment les proportions relatives de plantes en $C 3$ et de plantes en $C 4$. Hiernaux et al. (2006) montrent que cette composition et sa variation interannuelle peuvent influencer la production primaire des parcours au Sahel. Les simulations ne prennent pas en compte la texture et la fertilité des sols. Elles ne prennent pas en compte non plus les fluctuations des autres variables climatiques : rayonnement, température et humidité de l'air.

\section{Conclusion}

L'étude analyse la sensibilité de la production primaire de la végétation herbacée à la variation de la distribution intrasaisonnière des hauteurs de pluies locales. L'analyse des cycles saisonniers de pluies de trois stations pluviométriques situées à l'extrême nord, au centre et au sud de la région naturelle du Gourma au Mali, illustre l'importance du gradient d'aridité nord-sud observé dans la région. Les cumuls annuels de précipitations expliquent $74 \%$ de la variation interannuelle de la production aérienne herbacée sur le site d'Agoufou de 2002 à 2011. Cependant, les analyses de sensibilité réalisées par permutation des évènements de pluies au cours de la saison, révèlent que la relation entre les cumuls de pluies et la phytomasse herbacée masque des chutes de production intrasaisonnière liées aux stress hydriques notamment au cours de la période de croissance rapide et qui peuvent affecter la production annuelle. La faible différence entre les masses simulées et celles mesurées sur le site pastoral d'Agoufou, montre la capacité du modèle STEP à simuler la production primaire de la strate herbacée avec la pluie comme principal facteur limitant. Le modèle pourrait également servir dans un système intégré d'alertes précoces pour anticiper les pénuries de fourrage fréquentes dans cette région.

\section{CONFLITS D'INTERÊTS}

Les auteurs déclarent qu'il n'existe aucun conflit d'intérêts.

\section{CONTRIBUTIONS DES AUTEURS}

Les collègues co-auteurs PH, EM et NS ont participé à la collecte des données de phytomasse herbacée et de pluies dans le cadre des activités de l'Observatoire AMMA$\mathrm{CATCH}$ au Mali. Ils ont également contribué à l'analyse des données comme SS, AB et HSD.

\section{REMERCIEMENTS}

Les auteurs remercient les chercheurs et techniciens de l'Observatoire AMMA-CATCH au Mali. Les auteurs souhaitent également remercier les relecteurs pour leurs nombreux commentaires et suggestions.

\section{REFERENCES}

Achard F, Hiernaux P, Banoin M. 2001. Les jachères fourragères naturelles et améliorées en Afrique de l'Ouest. In $\mathrm{La}$ Jachère En Afrique Tropicale, Floret Ch, Pontanier R (eds). John Libbey Eurotext : Paris; 201-239. https://horizon.documentation.ird.fr/exldoc/pleins_textes/divers0903/010024637.pdf

Balme M, Lebel T, Amani A. 2006. Années sèches et années humides au Sahel : quo vadimus? J. Sci. Hydrol., 51(2) : 254271. DOI : $10.1623 /$ hysj.51.2.254

Balme M, Galle S, Lebel T. 2005. Démarrage de la saison des pluies au Sahel: variabilité aux échelles hydrologique et agronomique, analysée à partir des données EPSAT-Niger. Sécheresse, 16(1): 15-22. DOI : https://pdfs.semanticscholar.org/605d/3d 2d903f93119190b4d85c57e07f8aa39478 .pdf 
Delon C, Mougin E, Serça D, Grippa M, Hiernaux P, Diawara M, Galy-Lacaux C, Kergoat L. 2015. Modelling the effect of soil moisture and organic matter degradation on biogenic NO emissions from soils in Sahel rangeland (Mali). Biogeosciences, 12. DOI: 10.5194/bg-123253-2015

Diawara MO, Hiernaux P, Mougin E, Grippa M, Delon C, Diakité HS. 2018. Effets de la pâture sur la dynamique de la végétation herbacée au Sahel (Gourma, Mali): une approche par modélisation. Cahiers Agricultures., 27 : 15010. DOI : 10.1051/cagri/2018002

Diawara MO. 2015. Impact de la Variabilité Climatique au Nord Sahel (Gourma, Mali) sur la Dynamique des Ressources Pastorales, Conséquences sur les Productions Animales. Ph.D. Thesis, Université Toulouse 3, Toulouse, France, 176p.

http://www.theses.fr/2015TOU30029

Frappart F, Hiernaux P, Guichard F, Mougin E, Kergoat L, Arjounin M, Lavenu F, Koite M, Paturel J-E, Lebel T. 2009. Rainfall regime over the Sahelian climate gradient in the Gourma, Mali. Journal of Hydrology, 375 (1 - 2): 128 -142. DOI: 10.1016/j.jhydrol.2009.03.007

Hiernaux P, Diawara MO, Kergoat L, Mougin É. 2015. La contrainte fourragère des élevages pastoraux et agro-pastoraux du Sahel. In book: Les sociétés rurales face aux changements climatiques et environnementaux en Afrique de l'Ouest. Editors:

IRD https://www.researchgate.net/publication /303372607_

Hiernaux P, Mougin E, Diawara M, Soumaguel N, Diarra L. 2013. How much does grazing contribute to herbaceous decay during the dry season in Sahel rangelands? Élevage Climat et Société, ECliS 3.4, Géosciences Environnement Toulouse, GET, Toulouse, France, 22p.
Hiernaux P, Mougin E, Soumaguel N, Diawara M, Auda Y, Le Dantec V. 2013. Do seasonal maximum growth rates explain the large variations in annual yields of the herbaceous in Sahel rangelands? Doc. Trav. ECLIS, GET, Toulouse, 27p. https://www.researchgate.net/publication /318463481

Hiernaux P, Mougin E, Diarra L, Soumaguel N, Lavenu† F, Tracol Y, Diawara M. 2009. Rangeland response to rainfall and grazing pressure over two decades: herbaceous growth pattern, production and species composition in the Gourma, Mali. Journal of Hydrology, 375 (1-2): 114-127. DOI: 10.1016/j.jhydrol.2008.11.005

Hiernaux P, Le Houérou HN. 2006. Les parcours du Sahel. Sécheresse., 17: 51 71.

https://rmportal.net/framelib/parcourssahel.pdf

Jarlan L, Mangiarotti S, Mougin E, Hiernaux P, Mazzega P, Le Dantec V. 2008. Assimilation of SPOT/VGT-NDVI data into a sahelian grassland model. Remote Sens. Environ., 112: 1381-1394. https://www.documentation.ird.fr/hor/fdi :010042570

Mougin E, Diawara MO, Soumaguel N, Maïga AA., Demarez V, Hiernaux P, Grippa M, Chaffard V, Ba A. 2019. A leaf area index data set acquired in Sahelian rangelands of Gourma in Mali over the 2005-2017 period. Earth Syst. Sci. Data., 11: 675686. DOI : 10.5194/essd-11-675-2019

Mougin E, Hiernaux P, Kergoat L, Grippa M, de Rosnay P, Timouk F, Le Dantec V. 2009. The AMMA-CATCH Gourma Observatory site in Mali: relating climatic variations to changes in vegetation, surface hydrology, fluxes and natural resources. Journal of Hydrology, 375(162): 14-33. DOI: 10.1016/j.jhydrol.2009.06.045

Mougin E, Lo Seen D, Rambal S, Gaston A, Hiernaux P. 1995. A Regional Sahelian 
Grassland Model to be coupled with multispectral satellite data. I: Model description and validation. Remote Sens. Environ, 52:181-191.

Ousseina S, Fortina R, Marichatou H, Tenikoye A. 2013. Dynamique du peuplement herbacé de la station sahélienne expérimentale de Toukounous (Filingué - Niger). Int. J. Biol. Chem. Sci., 7(2): 657-671. DOI : 10.4314/ijbcs.v7i2.22

Pierre C, Bergametti G, Marticorena B, Mougin E, Lebel T, Ali A. 2011. Pluriannual comparisons of satellitebased rainfall products over the Sahelian belt for seasonal vegetation modeling. $J$. Geophys. Res., 116: D18201. DOI:10.1029/2011JD016115, 2011

Samandoulgou Y, Compaore H, Zoundi SJ, Zoungrana-Kabore YC. 2019. Evaluation de la productivité des herbacées fourragères des forêts sacrées de Koupéla dans le Centre Ouest du Burkina Faso. Int. J. Biol. Chem. Sci., 13 (1): 99. DOI : 10.4314/ijbcs.v13i1.9

Tracol Y. 2004. Etude des variations interannuelles de la production herbacée des pâturages sahéliens: exemple du Gourma Malien, Thèse Doct, SC Université Toulouse III, Paul Sabatier, 270p.

https://pdfs.semanticscholar.org/3486/9f e82a94c3d8d93f733f8c7b8f8d1b799f28. pdf

Tracol Y, Mougin E, Hiernaux P, Jarlan L. 2006. Testing a Sahelian grassland functioning model against herbage mass measurements. Ecological Modeling, 193(3-4): 437-446. DOI: 10.1016/j.ecolmodel.2005.08.033 